\title{
A note on the topological transversality theorem for the ad- missible maps of Gorniewicz
}

\section{Donal O’Regan}

School of Mathematics, Statistics and Applied Mathematics, National University of Ireland, Galway, Ireland.

\begin{abstract}
In this paper we discuss essential maps and the topological transversality theorem for maps admissible with respect to Gorniewicz.
\end{abstract}

Keywords: Essential maps, homotopy.

2010 MSC: 54H25, 55M20.

(C)2019 All rights reserved.

\section{Introduction}

The topological transversality theorem was established by Granas [6] for single valued maps. It was extended by many authors for Kakutani maps [9, 12], acyclic maps [2] and other general classes of maps $[3,10,11]$. In this paper we consider the topological transversality theorem for the admissible maps of Gorniewicz [4] and we obtain an "almost" topological transversality theorem. Our results improve those in [9] and we discuss briefly how one could obtain the "full" topological transversality theorem. We also discuss another approach to overcome the difficulty. One of the advantages of using the admissible maps of Gorniewicz (in comparison to using acyclic maps) is that the composition of maps admissible with respect to Gorniewicz are admissible with respect to Gorniewicz [4, 5].

For the remainder of this section we present the maps. Let $\mathrm{H}$ be the Čech homology functor with compact carriers and coefficients in the field of rational numbers $\mathrm{K}$ from the category of Hausdorff topological spaces and continuous maps to the category of graded vector spaces and linear maps of degree zero. Thus $H(X)=\left\{H_{q}(X)\right\}$ (here $X$ is a Hausdorff topological space) is a graded vector space, $H_{q}(X)$ being the q-dimensional Čech homology group with compact carriers of $X$. For a continuous map $f: X \rightarrow X, H(f)$ is the induced linear map $f_{\star}=\left\{f_{\star q}\right\}$ where $f_{\star q}: H_{q}(X) \rightarrow H_{q}(X)$. A space $X$ is acyclic if $X$ is nonempty, $H_{q}(X)=0$ for every $q \geqslant 1$, and $H_{0}(X) \approx K$.

Let $X, Y$ and $\Gamma$ be Hausdorff topological spaces. A continuous single valued map $p: \Gamma \rightarrow X$ is called a Vietoris map (written $p: \Gamma \Rightarrow X$ ) if the following two conditions are satisfied:

Email address: donal .oregan@nuigalway.ie (Donal O’Regan)

doi: $10.22436 /$ jnsa.012.06.01

Received: 2018-09-28 Revised: 2018-10-30 Accepted: 2018-12-01 
(i) for each $x \in X$, the set $p^{-1}(x)$ is acyclic;

(ii) $p$ is a perfect map i.e. $p$ is closed and for every $x \in X$ the set $p^{-1}(x)$ is nonempty and compact.

A map $\phi: X \rightarrow K(Y)$ is called an admissible map of Gorniewicz [4] (and we say $\phi \in \operatorname{Ad}(X, Y)$ ) provided there exists a space $\Gamma$ and two continuous maps $p: \Gamma \rightarrow X$ and $q: \Gamma \rightarrow X$ such that $p$ is a Vietoris map and $\phi(x)=q p^{-1}(x)$ for any $x \in X$; here $K(Y)$ denotes the family of nonempty compact subsets of $Y$. Note admissible maps of Gorniewicz are upper semicontinuous (u.s.c.).

\section{Essential maps}

Let $E$ be a topological space and $U$ an open subset of $E$.

Definition 2.1. We say $F \in A(\bar{U}, E)$ if $F: \bar{U} \rightarrow K(E)$ is a (u.s.c.) admissible compact map (i.e. $F: \bar{U} \rightarrow K(E)$ is a compact map and $F \in A d(\bar{U}, E))$; here $\bar{U}$ denotes the closure of $U$ in $E$.

Definition 2.2. We say $F \in A_{\partial u}(\bar{U}, E)$ if $F \in A(\bar{U}, E)$ and $x \notin F(x)$ for $x \in \partial U$; here $\partial U$ denotes the boundary of $U$ in $E$.

Definition 2.3. Let $F \in A_{\partial u}(\bar{U}, E)$. We say $F$ is essential in $A_{\partial u}(\bar{U}, E)$ if for every map $J \in A_{\partial u}(\bar{U}, E)$ with $\left.\mathrm{J}\right|_{\partial \mathrm{u}}=\left.\mathrm{F}\right|_{\partial \mathrm{u}}$ there exists a $x \in U$ with $x \in J(x)$. Otherwise $F$ is inessential in $A_{\partial u}(\bar{U}, E)$ i.e. there exists a map $\mathrm{J} \in \mathrm{A}_{\partial \mathrm{u}}(\overline{\mathrm{U}}, \mathrm{E})$ with $\left.\mathrm{J}\right|_{\partial u}=\left.\mathrm{F}\right|_{\partial u}$ and $x \notin J(x)$ for all $x \in \overline{\mathrm{U}}$.

Definition 2.4. Let $F, G \in A_{\partial u}(\bar{U}, E)$. We say $F \cong G$ in $A_{\partial u}(\bar{U}, E)$ if there exists a u.s.c compact map $\mathrm{H}: \overline{\mathrm{U}} \times[0,1] \rightarrow \mathrm{K}(\mathrm{E})$ with $\mathrm{H}(., \eta().) \in \operatorname{Ad}(\overline{\mathrm{U}}, \mathrm{E})$ for any continuous function $\eta: \overline{\mathrm{U}} \rightarrow[0,1]$ with $\eta(\partial \mathrm{U})=0, x \notin \mathrm{H}_{\mathrm{t}}(\mathrm{x})$ for any $\mathrm{x} \in \partial \mathrm{U}$ and $\mathrm{t} \in(0,1)$ (here $\left.\mathrm{H}_{\mathrm{t}}(\mathrm{x})=\mathrm{H}(\mathrm{x}, \mathrm{t})\right), \mathrm{H}_{0}=\mathrm{F}$ and $\mathrm{H}_{1}=\mathrm{G}$.

\section{Remark 2.5.}

(i) We do not assume $\cong$ is an equivalence relation in $A_{\partial u}(\bar{U}, E)$.

(ii) It is very easy to replace "compact maps" with " $k$-set contractive maps" (here $0 \leqslant k<1$ ) [9] or even " $k$-set countably contractive maps" (here $0 \leqslant k<1$ ) [1] in Definition 2.1 and 2.4 and obtain the obvious analogue of the results in this paper.

(iii) It is also very easy to replace "essential" with " $\Phi$-essential" [9-11] and obtain the analogue of the results in this paper (the obvious details are left to the reader).

We begin with the topological transversality theorem $[6,9,10]$.

Theorem 2.6. Let $\mathrm{E}$ be a Hausdorff topological vector space, $\mathrm{U}$ an open subset of $\mathrm{E}$ and suppose $\mathrm{F} \in \mathrm{A}_{\partial \mathrm{u}}(\overline{\mathrm{U}}, \mathrm{E})$. Then the following are equivalent:

(i) $F$ is inessential in $A_{\partial u}(\bar{U}, E)$;

(ii) there exists a map $\mathrm{G} \in \mathrm{A}_{\partial \mathrm{u}}(\overline{\mathrm{U}}, \mathrm{E})$ with $\mathrm{F} \cong \mathrm{G}$ in $\mathrm{A}_{\partial \mathrm{u}}(\overline{\mathrm{U}}, \mathrm{E})$ and $\mathrm{x} \notin \mathrm{G}(\mathrm{x})$ for all $\mathrm{x} \in \overline{\mathrm{U}}$.

Proof. First we prove (i) implies (ii). Let $\mathrm{G} \in A_{\partial \mathrm{u}}(\overline{\mathrm{U}}, \mathrm{E})$ with $\left.\mathrm{G}\right|_{\partial u}=\left.\mathrm{F}\right|_{\partial u}$ and $x \notin \mathrm{G}(x)$ for all $x \in \overline{\mathrm{U}}$. Define the map $\mathrm{H}$ by

$$
H(x, t)=(1-t) F(x)+t G(x) .
$$

Note $\mathrm{H}_{0}=F, \mathrm{H}_{1}=\mathrm{G}$ and it is well known [5] that $\mathrm{H}(., \eta().) \in \operatorname{Ad}(\overline{\mathrm{U}}, \mathrm{E})$ for any continuous function $\eta: \overline{\mathrm{U}} \rightarrow[0,1]$. Note also that $\mathrm{H}: \overline{\mathrm{U}} \times[0,1] \rightarrow \mathrm{K}(\mathrm{E})$ is a u.s.c. compact map (recall the sum of two compact sets is compact and the scalar multiples of compact sets are compact). Also if $x \in \partial U$ and $t \in(0,1)$ then since $\left.\mathrm{G}\right|_{\partial \mathrm{u}}=\left.\mathrm{F}\right|_{\text {au }}$ we have $H_{\mathrm{t}}(\mathrm{x})=(1-\mathrm{t}) \mathrm{F}(\mathrm{x})+\mathrm{t} \mathrm{G}(\mathrm{x})=\mathrm{F}(\mathrm{x})$ so $\mathrm{x} \notin \mathrm{H}_{\mathrm{t}}(\mathrm{x})$. Thus $\mathrm{F} \cong \mathrm{G}$ in $A_{\partial \mathrm{u}}(\overline{\mathrm{U}}, \mathrm{E})$, so (ii) holds.

Next we prove (ii) implies (i). Suppose there exists a map $G \in A_{\partial u}(\bar{U}, E)$ with $F \cong G$ in $A_{\partial u}(\bar{U}, E)$ and $x \notin G(x)$ for all $x \in \overline{\mathrm{U}}$. Let $H: \overline{\mathrm{U}} \times[0,1] \rightarrow \mathrm{K}(\mathrm{E})$ be a u.s.c. compact map with $\mathrm{H}(., \eta().) \in \operatorname{Ad}(\overline{\mathrm{U}}, \mathrm{E})$ 
for any continuous function $\eta: \bar{u} \rightarrow[0,1]$ with $\eta(\partial u)=0, x \notin H_{t}(x)$ for any $x \in \partial u$ and $t \in(0,1)$, $\mathrm{H}_{0}=\mathrm{F}$ and $\mathrm{H}_{1}=\mathrm{G}$. Let

$$
\mathrm{D}=\{x \in \overline{\mathrm{U}}: \mathrm{x} \in \mathrm{H}(\mathrm{x}, \mathrm{t}) \text { for some } \mathrm{t} \in[0,1]\} .
$$

If $\mathrm{D}=\emptyset$ then in particular $x \notin \mathrm{H}(x, 0)=\mathrm{F}(\mathrm{x})$ for $x \in \overline{\mathrm{U}}$ so $\mathrm{F}$ is inessential in $A_{\partial \mathrm{u}}(\overline{\mathrm{U}}, \mathrm{E})$ (take $\mathrm{J}=\mathrm{F}$ in Definition 2.3). It remains to consider the case when $D \neq \emptyset$. Now a standard argument [9] guarantees that $D$ is closed. In fact $D$ is compact since $H: \bar{U} \times[0,1] \rightarrow K(E)$ is a compact map and $D \subseteq H(D \times[0,1])$. Next note $D \cap \partial U=\emptyset$ (since $x \notin H_{t}(x)$ for any $x \in \partial U$ and $t \in(0,1), H_{0}=F \in A_{\partial u}(\bar{U}, E)$ and $\left.\mathrm{H}_{1}=\mathrm{G} \in \mathrm{A}_{\partial \mathrm{u}}(\overline{\mathrm{U}}, \mathrm{E})\right)$ so there exists (recall topological vector spaces are completely regular) a continuous map $\mu: \overline{\mathrm{U}} \rightarrow[0,1]$ with $\mu(\partial \mathrm{U})=0$ and $\mu(\mathrm{D})=1$. Define a map $\mathrm{J}: \overline{\mathrm{U}} \rightarrow 2^{\mathrm{E}}$ by $\mathrm{J}(\mathrm{x})=\mathrm{H}(\mathrm{x}, \mu(\mathrm{x}))$. Note $J \in \operatorname{Ad}(\bar{U}, E)$ and $\left.J\right|_{\partial u}=\left.H_{0}\right|_{\partial u}=\left.F\right|_{\partial u}($ since $\mu(\partial U)=0$ ). Finally note if there exists a $x \in \bar{U}$ with $x \in J(x)=H_{\mu(x)}(x)$ then $x \in D$ so $\mu(x)=1$ i.e. $x \in J(x)=H(x, 1)=G(x)$, a contradiction. Thus $\mathrm{J} \in \mathrm{A}_{\partial \mathrm{u}}(\overline{\mathrm{U}}, \mathrm{E})$ and $\left.\mathrm{J}\right|_{\partial u}=\left.\mathrm{F}\right|_{\partial u}$ and $x \notin J(x)$ for $x \in \overline{\mathrm{U}}$. Consequently $F$ is inessential in $A_{\partial u}(\overline{\mathrm{U}}, \mathrm{E})$.

Remark 2.7. If in Definition 2.4 we assumed $\mathrm{H}: \overline{\mathrm{U}} \times[0,1] \rightarrow \mathrm{K}(\mathrm{E})$ is a compact admissible map then automatically $\theta()=.H(., \eta().) \in \operatorname{Ad}(\overline{\mathrm{U}}, \mathrm{E})$ for any continuous function $\eta: \overline{\mathrm{U}} \rightarrow[0,1]$ with $\eta(\partial \mathrm{U})=0$ since $\theta()=.\mathrm{H} \circ \tau($.$) where \tau: \overline{\mathrm{U}} \rightarrow \overline{\mathrm{U}} \times[0,1]$ is given by $\tau()=.(., \eta()$.$) .$

Remark 2.8. There are very general topological transversality theorems in the literature and we mention two general results obtained by the author in [10, Theorem 2.14] and in [11, Theorem 2.1]; we remark here that in these two results the assumption that $\cong$ is an equivalence relation in $A_{\partial u}(\bar{U}, E)$ is not needed.

Remark 2.9. If we change the definition of an admissible map (i.e. change the definition of a morphism and spaces as in $[4$, Section 46$]$ or as in $[7,8])$ then in fact $\cong$ is an equivalence relation in $A_{\partial u}(\bar{U}, E)$ so in this case one would have immediately the "full" topological transversality theorem i.e. if $F$ and $G$ are two maps in $A_{\partial u}(\bar{U}, E)$ with $F \cong G$ in $A_{\partial u}(\bar{U}, E)$ then $F$ is essential in $A_{\partial u}(\bar{U}, E)$ if and only if $G$ is essential in $A_{\partial u}(\bar{U}, E)$. This point was inadvertently overlooked in [9, Theorem 2.8].

For the maps considered in this paper we will approach it differently and provide a partial result (see Corollary 2.12).

Theorem 2.10. Let $\mathrm{E}$ be a completely regular topological space, $\mathrm{U}$ an open subset of $\mathrm{E}, \mathrm{F} \in \mathrm{A}_{\partial \mathrm{u}}(\overline{\mathrm{U}}, \mathrm{E})$ and $\mathrm{G} \in \mathrm{A}_{\partial \mathrm{u}}(\overline{\mathrm{U}}, \mathrm{E})$ is essential in $\mathrm{A}_{\partial \mathrm{u}}(\overline{\mathrm{U}}, \mathrm{E})$. Also assume for any map $\mathrm{J} \in \mathrm{A}_{\partial \mathrm{u}}(\overline{\mathrm{U}}, \mathrm{E})$ with $\left.\mathrm{J}\right|_{\partial \mathrm{u}}=\left.\mathrm{F}\right|_{\partial u}$ we have $\mathrm{G} \cong \mathrm{J}$ in $\mathrm{A}_{\partial \mathrm{u}}(\overline{\mathrm{U}}, \mathrm{E})$. Then $\mathrm{F}$ is essential in $\mathrm{A}_{\partial \mathrm{u}}(\overline{\mathrm{U}}, \mathrm{E})$.

Proof. Consider any map $\mathrm{J} \in \mathrm{A}_{\partial \mathrm{u}}(\overline{\mathrm{U}}, \mathrm{E})$ with $\left.\mathrm{J}\right|_{\partial u}=\left.\mathrm{F}\right|_{\partial u}$. We must show there exists a $x \in \mathrm{U}$ with $x \in J(x)$. Let $H^{J}: \bar{U} \times[0,1] \rightarrow K(E)$ be a u.s.c. compact map with $H^{J}(., \eta().) \in A d(\bar{U}, E)$ for any continuous function $\eta: \overline{\mathrm{U}} \rightarrow[0,1]$ with $\eta(\partial \mathrm{U})=0, x \notin H_{t}^{J}(x)$ for any $x \in \partial U$ and $t \in(0,1)$ (here $\left.H_{t}^{J}(x)=H^{J}(x, t)\right), H_{0}^{J}=G$ and $H_{1}^{J}=J$ (this is guaranteed since $G \cong J$ in $A_{\partial u}(\bar{U}, E)$ ). Now let

$$
\Omega=\left\{x \in \overline{\mathrm{U}}: x \in \mathrm{H}^{\mathrm{J}}(\mathrm{x}, \mathrm{t}) \text { for some } \mathrm{t} \in[0,1]\right\} .
$$

Now $\Omega \neq \emptyset$ since $G$ is essential in $A_{\partial u}(\bar{U}, E)$ and $H_{0}^{J}=G$. A standard argument (since $H^{J}$ is u.s.c.) guarantees that $\Omega$ is closed and in fact it is compact (since $\Omega \subseteq \mathrm{H}^{\mathrm{J}}(\Omega \times[0,1])$ and $\mathrm{H}^{\mathrm{J}}$ is a compact map). Next notice $\Omega \cap \partial \mathrm{U}=\emptyset$ (since $x \notin H_{\mathrm{t}}^{J}(x)$ for any $x \in \partial U$ and $t \in(0,1), H_{1}^{J}=J \in A_{\partial u}(\bar{U}, E)$ and $\left.H_{0}^{J}=G \in A_{\partial U}(\bar{U}, E)\right)$ so there exists a continuous map $\mu: \bar{U} \rightarrow[0,1]$ with $\mu(\partial U)=0$ and $\mu(\Omega)=1$. Define the map $R$ by $R(x)=H^{J}(x, \mu(x))$. Now $R \in A \partial u(\bar{U}, E)$ with $\left.R\right|_{\partial u}=\left.G\right|_{\partial u}$ (note if $x \in \partial U$ then $R(x)=H^{J}(x, 0)=G(x)$ ). Since $G$ is essential in $A_{\partial u}(\bar{U}, E)$ there exists a $x \in U$ with $x \in R(x)$ (i.e. $\left.x \in H_{\mu(x)}^{J}(x)\right)$. Thus $x \in \Omega$ so $\mu(x)=1$. As a result $x \in H_{1}^{J}(x)=J(x)$.

Corollary 2.11. Let $\mathrm{E}$ be a completely regular topological space, $\mathrm{U}$ an open subset of $\mathrm{E}, \mathrm{G} \in \mathrm{A}_{\partial \mathrm{u}}(\overline{\mathrm{U}}, \mathrm{E})$ and $\mathrm{F} \in \mathrm{A}_{\partial \mathrm{u}}(\overline{\mathrm{U}}, \mathrm{E})$ is inessential in $\mathrm{A}_{\partial \mathrm{u}}(\overline{\mathrm{U}}, \mathrm{E})$. Also assume for any map $\mathrm{J} \in \mathrm{A}_{\partial \mathrm{u}}(\overline{\mathrm{U}}, \mathrm{E})$ with $\left.\mathrm{J}\right|_{\partial \mathrm{u}}=\left.\mathrm{F}\right|_{\partial \mathrm{u}}$ we have $\mathrm{G} \cong \mathrm{J}$ in $\mathrm{A}_{\partial \mathrm{u}}(\overline{\mathrm{U}}, \mathrm{E})$. Then $\mathrm{G}$ is inessential in $\mathrm{A}_{\partial \mathrm{u}}(\overline{\mathrm{U}}, \mathrm{E})$. 
Proof. Suppose $G$ is essential in $A_{\partial u}(\bar{U}, E)$. By assumption for any map $J \in A_{\partial u}(\bar{U}, E)$ with $\left.J\right|_{\partial u}=$ $\left.F\right|_{\partial u}$ we have $G \cong J$ in $A_{\partial u}(\bar{U}, E)$. Now Theorem 2.10 guarantees that $F$ is essential in $A_{\partial u}(\bar{U}, E)$, a contradiction.

Corollary 2.12. Let $\mathrm{E}$ be a Hausdorff topological vector space, $\mathrm{U}$ an open subset of $\mathrm{E}, \mathrm{F} \in \mathrm{A}_{\mathrm{\partial u}}(\overline{\mathrm{U}}, \mathrm{E})$ and $\mathrm{G} \in \mathrm{A}_{\partial \mathrm{u}}(\overline{\mathrm{U}}, \mathrm{E})$ is essential in $\mathrm{A}_{\partial \mathrm{u}}(\overline{\mathrm{U}}, \mathrm{E})$. Also assume $\mathrm{x} \notin \mathrm{tF}(\mathrm{x})+(1-\mathrm{t}) \mathrm{G}(\mathrm{x})$ for $\mathrm{x} \in \partial \mathrm{U}$ and $\mathrm{t} \in(0,1)$. Then $F$ is essential in $A_{\partial u}(\bar{U}, E)$.

Proof. Consider any map $\mathrm{J} \in \mathrm{A}_{\partial \mathrm{u}}(\overline{\mathrm{U}}, \mathrm{E})$ with $\left.\mathrm{J}\right|_{\partial \mathrm{u}}=\left.\mathrm{F}\right|_{\partial \mathrm{u}}$. We will show $\mathrm{G} \cong \mathrm{J}$ in $A_{\partial \mathrm{u}}(\overline{\mathrm{U}}, \mathrm{E})$ and then apply Theorem 2.10. Let

$$
H^{J}(x, t)=t J(x)+(1-t) G(x)
$$

Note $H_{0}^{J}=G, H_{1}^{J}=J, H^{J}: \bar{U} \times[0,1] \rightarrow K(E)$ is a u.s.c. compact map, and $H^{J}(., \eta().) \in \operatorname{Ad}(\bar{U}, E)$ for any continuous function $\eta: \overline{\mathrm{U}} \rightarrow[0,1]$. Also if $x \in \partial \mathrm{U}$ and $t \in(0,1)$ then since $\left.J\right|_{\partial u}=\left.\mathrm{F}\right|_{\partial \mathrm{u}}$ we have $H_{t}^{J}(x)=t J(x)+(1-t) G(x)=t F(x)+(1-t) G(x)$ so $x \notin H_{t}^{J}(x)$. Thus $G \cong J$ in $A_{\partial u}(\bar{U}, E)$. Now apply Theorem 2.10.

\section{References}

[1] R. P. Agarwal, D. O'Regan, Continuation theorems for countably condensing maps, Nonlinear Funct. Anal. Appl., 6 (2001), 1-18. 2.5

[2] R. P. Agarwal, D. O'Regan, A note on the topological transversality theorem for acyclic maps, Appl. Math. Lett., 18 (2005), 17-22. 1

[3] G. Gabor, L. Górniewicz, M. Ślosarski, Generalized topological essentiality and coincidence points of multivalued maps, Set-Valued Var. Anal., 17 (2009), 1-19. 1

[4] L. Górniewicz, Topological fixed point theory of multivalued mappings, Kluwer Academic Publishers, Dordrecht, (1999). 1, 2.9

[5] L. Górniewicz, M. Slosarski, Topological essentiality and differential inclusions, Bull. Austral. Math. Soc., 45 (1992), 177-193. 1, 2

[6] A. Granas, Sur la méthode de continuité de Poincaré, C. R. Acad. Sci. Paris, 282 (1976), 983-985. 1, 2

[7] W. Kryszewski, Topological and approximation methods of degree theory of set-valued maps, Dissertationes Math (Rozprawy Mat.), 336 (1994), 101 pages. 2.9

[8] W. Kryszewski, Homotopy properties of set-valued mappings, Uniwersytet Mikoaja Kopernika, Torun, (1997). 2.9

[9] D. O'Regan, Continuation methods based on essential and 0-epi maps, Acta Appl. Math., 54 (1998), 319-330. 1, 2.5, 2, $2,2.9$

[10] D. O'Regan, Generalized coincidence theory for set-valued maps, J. Nonlinear Sci. Appl., 10 (2017), 855-864. 1, 2, 2.8

[11] D. O'Regan, Topological transversality principles and general coincidence theory, An. Stiint. Univ. "Ovidius" Constanta Ser. Mat., 25 (2017), 159-170. 1, 2.5, 2.8

[12] R. Precup, On the topological transversality principle, Nonlinear Anal., 20 (1993), 1-9. 1 